\title{
Unknown structure of the Chephren pyramid
}

\author{
Janusz Kempa ${ }^{1, *}$, Wojciech Brylinski ${ }^{2}$ and Oskar Wyszynski ${ }^{3}$ for Pyramid Hunters ** \\ ${ }^{1}$ Warsaw University of Technology, Branch in Plock \\ ${ }^{2}$ Warsaw University of Technology, Faculty of Physics \\ ${ }^{3} \mathrm{CERN}$
}

\begin{abstract}
The Cheops and Chephren pyramid have been in Giza for about 3500 years. Their view is enjoyed by many people around the world who ask questions: how and why were the pyramids built? Cheops and Chephren pyramids are very similar on the outside. According to what we know, however, they are diametrically different in their internal structure. In the Chephren pyramid the internal structure was not discovered while the Cheops pyramid has many internal chambers, like: The King's Chamber, the Queen's Chamber. However, according to current knowledge, the Mummies of Pharaoh and his wife were never buried there. So, why were the internal structures of this pyramid built? Were the pyramids supposed to be a visible sign of Pharaoh's power? Are there any undiscovered chambers in both pyramids, which could give us answers to many questions?
\end{abstract}

\section{Introduction}

The internal structure of the Chephren pyramid was not discovered until now, except for one small room under the pyramid called the Balzoniz Chamber. To examine the pyramids internal structure, L. Alvarez placed detectors (spark chambers) in the Balzoniz Chamber [1]. After two years of measurements he selected around 25 thousand wakes of muons in vertical angles between $20^{\circ}$ and $40^{\circ}$. These measurements were compared to model calculations [2] (see Figure 1) for the expected stream of muons for different azimuthal angles from $0^{\circ}$ to $360^{\circ}$ assuming that the muon energy losses increase linearly with their energy $E_{\mu}$. The mathematical model of the Chephren pyramid was created for the presented calculations and can be easily adapted to examine the Cheops pyramid. For $\theta=$ $270^{\circ}$ an unexpectedly high peak was observed. This effect can be caused by irregularities in the construction or the presence of an undiscovered corridor. Between angles $\theta$ $=62^{\circ}$ and $\theta=72^{\circ}$ a possibly higher number of events in comparison to the model was observed. A possible explanation could be that a chamber is located there, which is similar in size to the King's Chamber in the Great Pyramid. Another area of interest is between $\theta=192^{\circ}$ and $\theta=$ $203^{\circ}$. An object of much higher density could explain the reduced number of muons.

\section{The experiment at Beamline for Schools}

To increase the understanding of muon interactions, a dedicated experiment was prepared at CERN [2]. The aim was to understand how the thickness of the limestone walls of

\footnotetext{
*e-mail: Janusz.Kempa@ pw.edu.pl

** B. Gutowski, W. Jozwiak, K. Komorowska, K. Krakowski, E. Pijus, K. Szymczak, M. Trojanowska - LO. Malachowianka, Plock, Poland
}

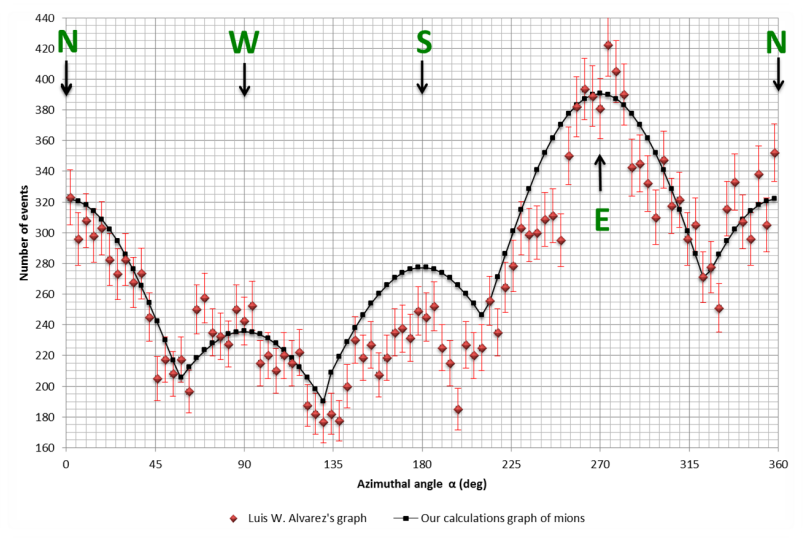

Figure 1. Results for 650.000 incident muons from zenith angles between $20^{\circ}$ and $40^{\circ}$. The calculations are normalized to the number of muons at $180^{\circ}$. Figure from ref. [2].

the pyramids affects the number of muons that penetrate them, for a given energy. The experimental setup is shown in Figure 2. The experiment was located at the CERN PS. Stones with the same geological properties as the Egyptian stones from which the pyramids are made were used. The purpose of the experiment was to measure muon attenuation for beam energies equal to $2.5 \mathrm{GeV}$ and $3 \mathrm{GeV}$. In this energy interval muon momentum losses are minimal. According to our best knowledge these are the only existing experimental results.

\subsection{Experimental details}

The incoming proton beam from the Proton Synchrotron accelerator impinges on the primary target and produces secondary particles, which enter the T9 experimental area. 


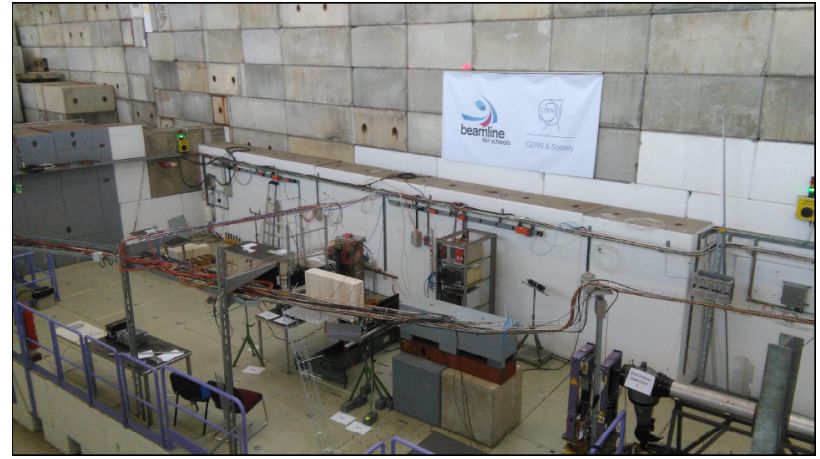

Figure 2. Panoramic photo of the experiment in the T9 hall. Figure from ref. [2].

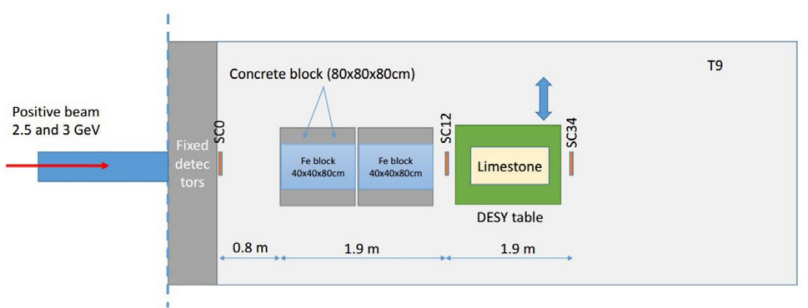

Figure 3. The schematic picture of the experiment in the T9 hall. Figure from ref. [2].

The collisions of the protons with the target provide a variety of particles, such as electrons, positrons, pions, kaons and (anti)protons. Muons are also present in the beam, from the decays of pions and kaons. The T9 beamline, used for the experiment, is therefore a mixed hadron and electron beam and can transport either positively or negatively charged particles with momenta between $0.5 \mathrm{GeV} / \mathrm{c}$ and $10 \mathrm{GeV} / \mathrm{c}$. The beam arrives in bursts of $4 \mathrm{~s}$. Depending on the scheduling, such a burst is provided typically once or twice per minute. The maximum number of particles per burst of $10^{6}$ is achieved for a $10 \mathrm{GeV} / \mathrm{c}$ positive beam and drops for lower momenta. For a negative beam, the rates are typically lower. The beam travels approximately $55 \mathrm{~m}$ before it enters the experimental area. The fixed setup of detectors consists of two Cherenkov counters, one scintillator and one delay wire chamber (DWC). The schematic picture of the experimental setup is presented on Figure 3.

The muon beam exiting the iron block is defocused and has a wide momentum distribution. The muon momentum is reduced by an average of approximately 1 $\mathrm{GeV} / \mathrm{c}$ for $80 \mathrm{~cm}$ of iron. After the muon filter, two scintillators in coincidence with a distance of $2.7 \mathrm{~m}$ between them count the number of muons entering the limestone. The limestone was positioned on a movable table. After the limestone, another scintillator coincidence measures the muons that passed through the entire thickness of the limestone.

The experiment at CERN allowed us to obtain an experimental picture of Landau fluctuations of muons with

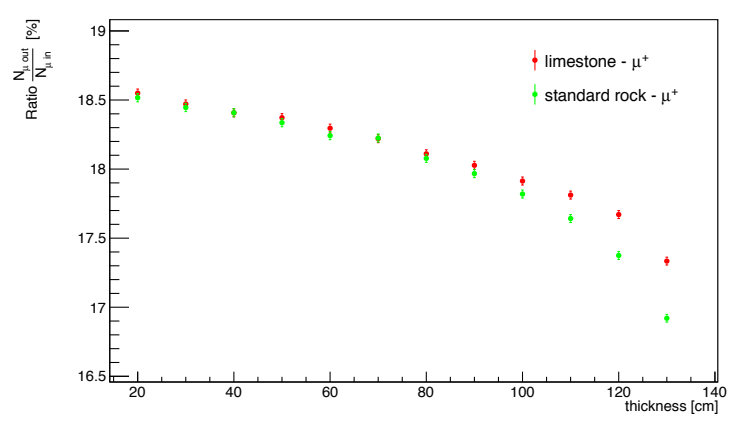

Figure 4. Ratio of muons that passed through the limestone over the initial number of muons, as a function of thickness of limestone for an energy of $3 \mathrm{GeV}$. The result includes the absorption of muons in the rock as well as muon scattering effects. The geometry was close to the experimental one [2].

different momenta passing through the limestone (details can be found in [2]).

\section{Calculations of muon attenuation in limestone}

Geant 4 was used to calculate how the muons pass through the limestone in the Chephren pyramid. The differences in the absorption of muons by standard rock $(\mathrm{A}=22, \mathrm{Z}=$ $11, \rho=2.65 \mathrm{~g} / \mathrm{cm}^{3}$ ) and by limestone rock were observed. The numbers of muons passing through successive rock layers from $20 \mathrm{~cm}$ to $130 \mathrm{~cm}$ were determined. In addition, it was calculated how many muons are passing through $500 \mathrm{~cm}$ of rock. A plot of the ratio of muons that passed through the limestone over the initial number of muons with respect to the rock thickness can be seen in Figure 4 for the initial muon energy of $3 \mathrm{GeV}$.

The results presented in Figure 4 show the differences in the absorption of muons in the standard rock and in the limestone rock from which the pyramids of Giza were built. In limestone rocks, muons are absorbed more slowly than in standard rock. This is due to the difference in the chemical composition of these rocks and their density. Limestone rocks have a density smaller than standard rock by about $0.4 \mathrm{~g} / \mathrm{cm}^{3}$. In addition, the number (percent) of passing muons by the 5-meter layer of limestone is equal to $(0.0999 \pm 0.0032) \%$ and for standard rock $(0.0322 \pm$ $0.0018) \%$.

The obtained result is essential to determine the threshold energy of cosmic ray muons that pass through the rocks and reach the muon detector. Considering this effect and the fluctuation of the muon range, it allows to calculate the number of cosmic ray muons arriving to the detectors.

\section{Discussion and conclusions}

Interest in the pyramids of Giza is not diminishing. Egyptian culture is part of a world culture, extremely important and still not fully understood. 
That is why, with the greatest curiosity, the most recent information about the discovery in the Cheops pyramid of an unknown chamber located above the Grand Gallery was accepted [3]. This chamber, recently discovered, enriches the internal structure of the Cheops pyramid (see Figure 5). We are waiting for information about the purpose of the newly discovered chamber. The rich internal structure of the Cheops pyramid increases the interest in the Chephren pyramid. Could it be that Chephren, son of Cheops, changed the architecture of the pyramid in a short time? Where were the mummies of Cheops and Chephren buried? We wait with the hope that someone will make once again a muon tomography of Chephren's pyramid.

At the same time, we showed in this work that when interpreting the measurement results of cosmic muon absorption, the chemical composition of the limestone rocks from which the pyramids in Giza were constructed should be correctly taken into account. The range of muons in these rocks is significantly larger than in the standard rocks. The range of muons with energy $2.5 \mathrm{GeV}$ in standard rocks with good accuracy is equal to about $5 \mathrm{~m}$.

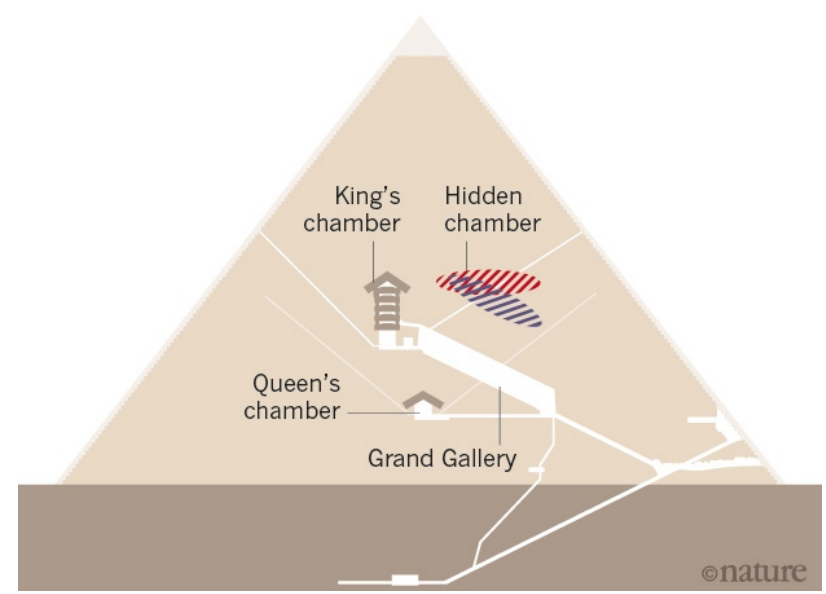

Figure 5. Current image of the internal structure of the Cheops pyramid. Figure from ref. [3].

\section{References}

[1] Alvarez, L. W. et al., Science 167 (3919), 832-839

[2] Gutowski B. et al., Phys. Educ. 53 (2018) 045011 (8pp)

[3] Morishima K. et al. Nature 552, 386-390 (2017). 